University of San Diego

Digital USD

Spring 5-26-2018

\title{
In honor of self: A weight loss project for a military population with mental disorders
}

Tanya Denise Stevenson-Gaines

University of San Diego, tsgnav05@yahoo.com

Follow this and additional works at: https://digital.sandiego.edu/dnp

Part of the Nursing Commons, and the Social and Behavioral Sciences Commons

\section{Digital USD Citation}

Stevenson-Gaines, Tanya Denise, "In honor of self: A weight loss project for a military population with mental disorders" (2018). Doctor of Nursing Practice Final Manuscripts. 77.

https://digital.sandiego.edu/dnp/77

This Doctor of Nursing Practice Final Manuscript is brought to you for free and open access by the Theses and Dissertations at Digital USD. It has been accepted for inclusion in Doctor of Nursing Practice Final Manuscripts by an authorized administrator of Digital USD. For more information, please contact digital@sandiego.edu. 
In Honor of Self: A Weight Loss Project for a Military Population with Mental Disorders

Authors: Tanya D. Stevenson-Gaines and Kathy James

Corresponding author

Tanya D. Stevenson-Gaines, MSN

Doctor of Nursing Practice (DNP) PMHNP Student, USD Hahn School of Nursing and Health Science

Complete mailing address:

13988 Royal Melbourne Square

San Diego, CA 92128-3646

Business Phone: 1-858-444-7007

Email: tstevensongaines@ @andiego.edu

Co-Author

Kathy James, DNSc, APRN, FAAN,

Professor, USD Hahn School of Nursing and Health Science, San Diego, CA, USA

Conflicts of Interest: Tanya D. Stevenson-Gaines and Kathy James declare no conflicts of interest with the research or writing of this paper.

Funding Statement: This project was funded by the student.

Author Contributions: TSG conceived the study and determined the methodology. TSG collected and analyzed the data that was not entered into a statistical program. Both authors reviewed the findings. TSG took the lead in writing and organizing the manuscript. TSG wrote the background and method sections, and KJ edited the sections as a subject matter on weight loss. Both authors reviewed the final manuscript before submitting for publication.

Other Acknowledgments: Tanya D. Stevenson-Gaines would like to thank the leaders in the military treatment facility (MTF) where the data was collected for their support. Thank you Dr. Donna Agan for your editorial assistance, and Dr. Robert Riffenburgh for your statistical analysis of the project data. 
In Honor of Self: A Weight Loss Project for a Military Population with Mental Disorders

Abstract

Background: Obesity in people diagnosed with mental disorders is at an epidemic level. Individuals with a mental illness who are prescribed medication for stability are at risk for obesity and metabolic syndrome. People with mental disorders have a 10 to 30 -year decrease in life expectancy associated with chronic illnesses. Military personnel are separated from service when they are measured outside of body composition assessment standards.

Aims: To address the problem of obesity in military members by providing a weight loss program with structured and collaborative weight management education and telephonic coaching.

Methods: Stakeholders were informed about the purpose and goals for the intervention. The project timeline was 3 to 6 months. Participants attended an integrated nutrition and exercise class and were encouraged to maintain food intake and exercise journals. Participant weight, BMI, daily intake of fruits and vegetables, and exercise activity were monitored. Follow-up telephonic coaching was provided.

Results: Ten subjects participated in the program. One individual dropped in December 2017. Seven answered their telephone in February 2018. Twenty percent of the participants lost 5\% of their baseline weight, $43 \%$ increased their daily intake of fruits and vegetables, and 57\% increased their exercise activity. The group average for exercise time per week increased from 66 to 146 minutes.

Conclusions: A weight loss program can be safe, beneficial, and efficacious to assist individuals with a mental disorder to lose weight.

Keywords: mental disorder, military, weight loss program 


\section{Introduction}

Obesity in mental health patients is at an epidemic level. Individuals with mental illness who are prescribed medication for stabilization and illness remission are at risk to develop obesity and metabolic syndrome. Metabolic syndrome can result in hypertension, type 2 diabetes, elevated blood cholesterol, and cardiovascular disease. The National Institute of Health (2013) reported that more than $80 \%$ of people with serious mental illnesses were overweight or obese. It was reported that patients with a mental disorder had a mortality rate three times greater than the general public and a 10 to 30-year decrease in life expectancy (Daumit et al., 2013). People treated in the behavioral health care environment are at risk of becoming obese. Additionally, mentally ill persons were reported to seek more weight assistance care than people without a mental disorder (Copeland, Pugh, Hicks, \& Noel, 2012). Patients diagnosed with schizophrenia, anxiety, or a bipolar condition were reported to have an elevated body mass index (BMI). A BMI of 32.5 also increased a patient's probability of developing metabolic syndrome. BMI for patients with Cluster A and Cluster B personality disorders were reported in the obese ranges. BMIs from 25-29 were considered overweight and 30 or greater were obese (Center for Disease Control and Prevention, 2016; Stanley, Laugharne, Addis, \& Sherwood, 2013). In 2006, the average cost to treat an obese individual was $\$ 1,429$ more than the cost of providing care for a person in the normal weight range (Harvard School of Public Health, n.d.).

Patients diagnosed with bipolar, major depression, anxiety, panic attack, major depression, and agoraphobia disorders report higher rates of obesity and patients with a bipolar disorder experienced the highest rate of obesity. Researchers have recommended that exercise and weight management be incorporated into long term behavioral health care planning.

Medications used to treat various mental disorders were also associated with weight gain, 
particularly the medication categories of anti-psychotics, selective serotonin receptor inhibitors (SSRIs), tricyclic antidepressants, monoamine oxidase inhibitors, Divalproex, lithium, and gabapentin (Gibson, Carek, \& Sullivan, 2011). Since the risk of developing metabolic syndrome is greater with BMIs above 25, the American Diabetes Association developed treatment guidelines and recommendations for health care providers to minimize weight gain through patient education, baseline screening, and frequent monitoring. The guidelines suggest how to reduce metabolic effects in patients with mental disorders (e.g., ethnicity assessment, BMI status, fasting blood glucose and lipid panel monitoring, assessing blood pressure > 140/90). The guideline recommends maintaining a BMI of less than 25 with low-calorie food intake, exercise, and behavior therapy for weight loss and maintenance. If weight loss is a goal, then the individual's weight loss efforts should be assessed after a 24-week period (Gibson, Carek, \& Sullivan, 2011).

Daumit, et al. (2013) reported that individuals with mental disorders could lose weight when provided with nutrition and exercise support and that a structured weight-loss program with nutrition and exercise instruction was safe and efficacious for these overweight and obese individuals. Other researchers reported that, within a population of 171,884 veterans, that posttraumatic stress disorder (PTSD) was associated with obesity and metabolic syndrome as comorbidity conditions. There was an association between PTSD and poorer exercise as well as dietary habits in military and civilian communities, Veterans reported poorer food choices, an intake of large amounts of food, fast eating, and hoarding of high caloric foods. That behavior may have resulted from decreased food choices in theatre with behaviors continuing post deployment. Increased stress can also result in emotional eating in individuals with PTSD 
(Klingaman et al., 2016). Additionally, therapeutic actions and side effects of the medication can create a desire to eat, and a change in glucose metabolism.

\section{Evidence for the Problem}

A literature search utilized the search engines CINAHL, Pubmed, Psych Info, and the Cochrane database. Key words used for article searches were mental illness, obesity, and weight loss. To narrow the search for literature related to the project, abstracts were initially reviewed for relevance. If the abstract significantly addressed activity concepts relevant to the problem and the EBP activities, the full article was read to confirm usefulness of the reference. Interlibrary loan procedures were utilized to request retrieval of five full-text references. Twenty-one references were reviewed and 15 were selected for potential use.

There were six evidence-based practice (EBP) strategies implemented to accomplish the overall project goals. The first strategy was to schedule a meeting to educate key stakeholders (e.g., mental health leaders, providers) as well as facility health and wellness leaders and staff about the literature regarding obesity and mental health treatment as well as the proposed project. Meeting with the key stake holders and department providers was crucial to receiving buy-in and encouraging feedback about the project and implementation plans. Cabassa et al. (2011) recommended the incorporation of exercise, nutrition, and health promotion into the medical treatment plan as a way to accomplish a decreased incidence of excess weight and obesity in patients with mental disorders, to improve recovery, to decrease disease co-morbidity, to prevent premature death, and to identify health care cost savings from the intervention efforts (Cabassa, Ezell, \& Lewis-Fernández, 2010; Verhaeghe, De Maeseneer, Maes, Van Heeringen, \& Annemans, 2011). 
The second activity-related EBP strategy involved the duration of the intervention. The project was scheduled to last for 3 to 6 months. Ideal time for weight loss assessment ought to be 6-months. Researchers reported ideal participant weight loss of at least $5 \%$ of baseline body weight by that time as a weight loss of at least $5 \%$ of body weight provided a demonstrable health benefit (Daumit et al., 2013; Green et al., 2015). The third EBP-related activity involved the provision of exercise education. To ensure and promote patient safety, all participants were required to have an exercise consultation entered into their electronic health record (EHR) prior to being scheduled to receive information about any recommended physical activity (Green et al., 2015; Temmingh et al., 2013). The fourth EBP-related activity involved the provision of nutrition education. The participants attended a 2-hour, integrated nutrition and exercise course. Participants were scheduled to attend the course either by mental health provider consultation or by email notification to the DNP student that they expressed an interest to enroll in the project. Literature recommended that nutritional education topics include caloric intake, minimizing high sugar content food, eating at least five servings of fruits and vegetables daily, and adequate daily beverage consumption (Daumit et al., 2013; Green et al., 2015). Dietary education recommended that participants receive instruction on reading food labels, keeping a food log, and the importance of observing the amount of food consumed (Cabassa et al., 2013). During the 2-hour integrated nutrition and exercise courses, the DNP student provided food and exercise logs for participants to use in the STRIDE weight loss and lifestyle intervention for individuals taking antipsychotic medications study with researcher permission (Green et al., 2015).

The fifth activity involved height and weight measurements to calculate the individual's BMI. To achieve the most accurate body height and weight, a morning weight was encouraged utilizing the same scale without shoes. If attainment of a morning weight was not feasible, an 
afternoon height and weight measurement was accepted (Daumit et al., 2013; Verhaeghe et al., 2011). The sixth EBP activity for this project involved follow-up telephone calls to the participants. The student attempted to contact each participant by telephone weekly for the first month, then monthly for the additional 2 months. During the telephone conversation, the participant was asked about the number of servings of fruits and vegetables consumed daily, days of exercise each week, and minutes of exercise performed weekly. They were queried for questions, barriers, and the need for support. If barriers were noted, the DNP student assisted the individual to resolve the issue by providing alternative, healthy lifestyle solutions mentioned in literature or offered to assist them in contacting the dietician or exercise trainer providing instruction during the integrated nutrition and exercise course. Literature reported that wellness coaching provided by telephone resulted in a significant amount of weight loss in individuals diagnosed with a mental disorder (Green et al., 2015; Temmingh et al., 2013).

\section{Framework}

The Ottawa Model of Research Use (OMRU) was the EBP model selected for use in this weight loss intervention project. This model offered steps that were easy to follow as well as clear and prescriptive steps ideal and achievable in a military health care setting. The model promoted patient involvement and addressed project turnover that was essential for ownership, commitment, and sustainment. The six elements of the model were utilized as a framework to plan, initiate, implement, and assess the outcome measures (Rycroft-Malone, 2010). The purpose of the weight loss program was to address the problem of obesity in military members and their families receiving mental health treatment by providing structured and collaborative weight management education followed by telephonic coaching. 


\section{Methods}

\section{Participants and Recruitment}

A program informational flyer was created by student. The flyer included graphic pictures for each component of the program on (a) nutrition education, (b) exercise instruction, (c) weight and BMI assessment, (d) telephone support, (e) a collaborative team to assist the patient, and (f) the reward for potential weight loss. The student's contact information; the project duration dates; and dates, times, and locations for the integrated exercise and nutrition courses were included. The flyer was distributed to mental health providers during in-person meetings as well as electronically. Project implementation occurred from the months of November 2016 through February 2018. Participants were required to be at least 18 years old, verbalize interest, and consent to enrollment in the weight loss program. Mental health providers submitted a consult for the participant to attend a nutrition and exercise course offered by the Health and Wellness Department. The student networked with health and wellness staff to create a consultation site in the electronic health record (EHR) to send referrals for easy identification and course booking. Participant recruitment began in October 2017, subsequent to facility and university IRB approvals. Ten participants voluntarily enrolled after they completed an integrated nutrition and exercise course.

Although one individual weighed approximately 60 pounds less than the average weight for the group, all individuals were identified to be overweight or obese according to their BMI measurement. All of the individuals were active duty members. There were five males and five females with ages ranging from their 20 s through 40 s. Six individuals were Caucasian. The other four participants were of African or Latino descent. 


\section{Project Design}

To promote safety, a medical provider was selected to review participants' health information for exercise before enrollment in the program. Information presented in the 2-hour nutrition and exercise course was delivered with PowerPoint slides, question and answer sessions, the use of exercise bands, and demonstration. Information on caloric intake, minimizing high sugar content food, eating at least five servings of fruits and vegetables daily, and beverage consumption was included. The student was present during the courses and was invited to emphasize literature recommendations on healthy eating and safe exercise. Refrigerator magnets were created with the student's business card and an apple sticker was placed on the upper left-hand corner. Participants were asked to place the magnet on or near their refrigerator to remind them that they were enrolled in the program. The exercise instructor illustrated how to safely use exercise bands for strength training and encouraged participants to practice the movements in class. Participants were encouraged to keep track of their fruits and vegetables intake and exercise activity using a log of their choice by hard copy document, an online resource, or on their cellphones. If they preferred, they were encouraged to purchase and use an exercise tracking device to monitor their fitness activity. Baseline weight and BMI measurements were collected for each participant for comparative analysis of weight loss over time. Monthly participant weight and BMI were collected from November 2017 through February 2018 by accessing their EHR. Benchmarks outcomes were established for the project to compare the results with literature findings. They included (a) $40 \%$ of the participants would lose $5 \%$ of their baseline weight, (b) $40 \%$ would eat 5 servings of fruits and vegetables daily, (c) $40 \%$ would exercise 5 days each week, and (d) $40 \%$ would exercise 150 minutes per week. 
The data on the number of daily servings of fruits and vegetables, days of exercise each week, and completed minutes of exercise per week were collected during telephone contact. That information was documented on a telephone script-form designed by the student to include the nutrition and exercise variables. The form was adapted from the Johns Hopkins Medicine web page with permission to modify the document (Johns Hopkins, 2008). Each telephone call began with an introduction of the student, the reason the participant was being contacted, and concluded with asking whether the individual had questions, a thank you, and a good-bye statement. The student established a time to call the participants by telephone; from 6:30 p.m. until 7:30 p.m. on a Friday. This practice allowed the participant to create a schedule that included the expected telephone call from the student without interruption to his or her daily routine. On occasion, participants scheduled another day and time for telephone contact due to unforeseen circumstances. A password-protected Microsoft Excel spreadsheet was designed by the student to document participant weight, BMI, nutrition, and exercise activity. The information was maintained on a Department of Defense computer with identifying (ID) numbers from 1 to 10 assigned to each participant to ensure privacy.

A SurveyMonkey satisfaction questionnaire was developed by the student with five openended questions to assess participant satisfaction after completion of the program. T-shirts and a participation thank-you card were offered to each participant with the approval of the facility's Staff Judge Advocate's Office.

\section{Data Analysis Procedure}

Data analysis was performed with the assistance of the military facility's biostatistician; the student emailed a password-protected Excel spreadsheet to the biostatistician. The biostatistician used the Stata (Version 13) program to analyze the data. With each project 
variable and follow-up, and contact date an analysis was made of the number of observations, mean, standard deviation, minimum and maximum number reported for each variable. Since the number of participants was small, the student hand-calculated data on the percent of the participants who (a) lost 5\% of their baseline weight, (b) ate five servings of fruits and vegetables daily, and (c) exercised 5 days and 150 minutes per week at the end of the project. For some time-points, participants were not available by phone and that resulted in an annotation of zero in the spreadsheet.

\section{Institutional Review Board}

The project was reviewed and approved by the facility research facilitator and institutional review boards in the military facility and the university. The project was identified as a quality improvement (QI) project rather than a research study: thereby, it was declared exempt by the respective institutional review boards. Throughout the implementation process, participants were informed that their identification would be protected. The military facility requested that raw data not be viewed outside of the facility and only generalized results of the project be reported.

\section{Results}

Ten participants voluntarily enrolled in the program. Their ages ranged from 20 s to 40 s. Five were male and five were female. Six individuals were Caucasian. The other four participants were of African or Latino descent. One male of African descent dropped out of the program in the month of December 2017. The ability to contact participants was inconsistent, although the student was able to consistently monitor monthly weights and BMI information documented in their EHR. Because of access to the EHR, the student was able to assess the participant's weight and BMI for those who dropped from the program. The initial study 
agreement with participants granted the student permission to monitor weight and BMI throughout the project. As shown in Table 1, 100\% of the participants had weight and BMI data in their EHRs pre-implementation and on the last measurement assessment in February 2018. In the month of December 2017, weight and BMI information were not documented for two participants. The one participant who dropped out of the program in December 2017 gained 21 pounds during the 3-month project; the largest weight gain for any participant. The next largest participant weight gain was 7 pounds. The large weight gain of the dropout participant affected the group average for weight and BMI measurements.

As illustrated in Table 1, 5 to 7 individuals were available by phone during each scheduled follow-up encounter for the group. From December 2017 through February 2018, seven scheduled telephone calls were for patient follow-up and coaching. For the group, fruits and vegetables increased from 3 to 4 servings daily, weekly exercise increased from 2 to 3 days, and time in weekly exercise increased from 66 minutes to 146 minutes. A SurveyMonkey satisfaction questionnaire was developed by the student with five open ended questions to measure participant satisfaction after completion of the program. Five participants completed the non-identified survey with the following results: (a) $100 \%$ of the respondents found the program to be helpful in assisting them to lose weight, (b) three individuals liked having to be accountable for their behavior with telephone follow up, and (c) three individuals endorsed eating more fruits and vegetables. Two people mentioned that they would have preferred to have more group support to work on their weight loss goals.

Table 2 provides information on the outcomes for the five variables compared with the pre-established project benchmarks. Two (20\%) of the participants lost 5\% of their baseline weight. Three additional individuals lost an average of 3 pounds. Three of the seven individuals 
who answered the phone in February 2018 reported eating five servings of fruits and vegetables daily. Therefore, $43 \%$ reported eating five servings of fruits and vegetables daily at the end of the project. One participant of the 7 program completers reported exercising 5 days per week. Therefore, $14 \%$ endorsed exercising 5 days per week by the end of the program. Four out of seven program completers reported exercising 150 minutes weekly. Therefore, $57 \%$ exercised at least 150 minutes at the end of the program. With those findings, the benchmark percentage goals for daily intake of fruits and vegetables and exercising 150 minutes each week were achieved. It is worthwhile to note that, although the project was implemented over a major holiday season, five participants were able to lose weight. There was no statistically-significant change in pre and post weight and BMI measurements (Table 1).

\section{Discussion}

\section{Summary}

A 3-month, structured, and collaborative weight loss program was implemented in a military health care setting with 10 active duty volunteers receiving treatment for mental disorders. Primary interventions were group nutrition and exercise education followed by telephone coaching. Variables were weight, BMI, daily intake of fruits and vegetables, days of exercise per week, and weekly minutes of exercise. Positive changes in some of the participants were observed in eating and exercise behaviors as well as in weight loss.

\section{Interpretation}

The project had comparable results as noted in literature; patients prescribed psychotropic medications for mental health treatment can lose weight when enrolled in a collaborative, structured, weight loss program. Direct health care costs to provide care to 10 individuals over normal weight were estimated to be $\$ 12,856$ ( $\$ 1,285.60$ per person). The literature reported an 
additional cost of $\$ 266$ to provide care for an overweight individual and an additional $\$ 1,723$ to provide care for an obese individual (Obesity Prevention Source, 2017). As three individuals were overweight $(\mathrm{BMI}>25)$ and seven were obese $(\mathrm{BMI}>30)$. The net program costs were estimated at \$698 (Center for Disease Control and Prevention, 2016). The projected program cost was calculated using the dollar amount to educate staff about the program according to their estimated hourly wages; the estimated cost to educate the participants was calculated using their estimated hourly wage. The cost for the intervention training conducted by the dietician and exercise instructor was calculated using their estimated hourly wage. The student's hourly wage to train staff about the program was also considered (Lu, Bakas, \& Haase, 2013). The amount of labor costs for telephonic patient contact was not calculated. The return on investment (ROI) was calculated based on every $\$ 1$ spent. Therefore, $\$ 17.42$ could be saved if the 10 participants could achieve a normal BMI measurement.

\section{Limitations}

There were fewer participants enrolled in the project than expected. The original planned project duration was for 6-months beginning in August 2017. In literature, the best change in healthy behaviors and weight loss outcomes were observed at 6-months (Daumit et al., 2013; Green et al., 2015). The IRB approval process was delayed due to staff changes. When the project was approved by the research facilitator, an additional 2 weeks was needed to get participants enrolled in the program. Conflicting responsibilities occurred with the staff assigned to review program consultations and contact patients for enrollment into the integrated nutrition and exercise course. The enrollment dates were extended 2-weeks to increase the number of participants enrolled. Three 2-hour classes were scheduled with a final enrollment number of 10 participants. Two of the classes had three individuals enrolled and one class had four 
participants enrolled. The program enrollment process improved when the student was notified about the patient consultations and called the participants to enrollment them in the program. Two patients contacted the student by email to communicate their interest in being enrolled in the program. There were approximately five individuals recommended to attend the program but could not because of personal and occupational responsibilities.

\section{Conclusions}

A weight loss program can be safe, beneficial, and efficacious to assist individuals with a mental disorder to lose weight. More research and EBP projects need to be done to optimize the health of individuals with mental health disorders. 


\section{References}

Cabassa, L. J., Ezell, J. M., \& Lewis-Fernández, R. (2010). Lifestyle interventions for adults with serious mental illness: A systematic literature review. Psychiatric Services, 61, 774782.

Center for Disease Control and Prevention. (2016). Defining adult overweight and obesity. Retrieved from https://www.cdc.gov/obesity/adult/defining.html

Copeland, L. A., Pugh, M. J., Hicks, P. B., \& Noel, P. H. (2012). Use of obesity-related care by psychiatric patients. Psychiatric Services, 63, 230-236.

Daumit, G. L., Dickerson, F. B., Wang, N. Y., Dalcin, A., Jerome, G. J., Anderson, C. A., . . . Appel, L. J. (2013). A behavioral weight-loss intervention in persons with serious mental illness. New England Journal of Medicine, 368, 1594-1602.

Gibson, M., Carek, P. J., \& Sullivan, B. (2011). Treatment of co-morbid mental illness in primary care: How to minimize weight gain, diabetes, and metabolic syndrome. International Journal of Psychiatry in Medicine, 41, 127-142.

Green, C. A., Yarborough, B. J. H., Leo, M. C., Stumbo, S. P., Perrin, N. A., Nichols, G. A., \& Stevens, V. J. (2015). Weight maintenance following the STRIDE lifestyle intervention for individuals taking antipsychotic medications. Obesity, 23, 1995-2001.

Harvard School of Public Health. (n.d.). Obesity Prevention Source:Economic costs. Retrieved from https://www.hsph.harvard.edu/obesity-prevention-source-source/obesityconsequences/economic/

Johns Hopkins Medicine. (2008). Sample telephone script. Retrieved from https://www.hopkinsmedicine.org/institutional/telephonescreeningscript.doc 
Klingaman, E. A., Hoerster, K. D., Aakre, J. M., Viverito, K. M., Medoff, D. R., \& Goldberg, R. W. (2016). Veterans with PTSD report more weight loss barriers than veterans with no mental health disorders. General Hospital Psychiatry, 39, 1-7.

Lu, Y. Y., Bakas, T., \& Haase, J. E. (2013). Cost template for meaningful activity intervention for mild cognitive impairment. Clin Nurse Spec, 27(2), 88-95.

National Institute of Health. (2013). Study shows people with serious mental illness can lose weight. Retrieved from https://www.nimh.nih.gov/news/science-news/2013/nih-studyshows-people-with-serious-mental-illness-can-lose-weight.shtml

Rycroft-Malone, J., \& Bucknall, T. (Eds.). (2010). Models and frameworks for implementing evidence-based practice: Linking evidence to action. Chichester, England: WileyBlackwell.

Stanley, S. H., Laugharne, J. D., Addis, S., \& Sherwood, D. (2013). Assessing overweight and obesity across mental disorders: personality disorders at high risk. Social Psychiatry and Psychiatric Epidemiology, 48, 487-492.

Temmingh, H., Claassen, A., van Zyl, S., Carrara, H., Dayakalashe, N. M., Myer, L., \& Stein, D. J. (2013). The evaluation of a telephonic wellness coaching intervention for weight reduction and wellness improvement in a community-based cohort of persons with serious mental illness. Journal of Nervous and Mental Disease, 201(11), 977-986.

Verhaeghe, N., De Maeseneer, J., Maes, L., Van Heeringen, C., \& Annemans, L. (2011). Effectiveness and cost-effectiveness of lifestyle interventions on physical activity and eating habits in persons with severe mental disorders: a systematic review. International Journal of Behavioral Nutrician and Physical Activity, 8, 28. 
Table 1

Participant Weight, BMI, Intake of Fruits and Vegetables and Exercise Behaviors from

December 2017 to February 2018

\begin{tabular}{|c|c|c|c|c|c|}
\hline & $n$ & $M$ & $S D$ & Minimum & Maximum \\
\hline \multicolumn{6}{|l|}{ BMI } \\
\hline Pre BMI & 10 & 32 & 4 & 26 & 36 \\
\hline Dec 17 BMI & 8 & 31 & 4 & 27 & 36 \\
\hline Jan 18 BMI & 10 & 32 & 3 & 26 & 36 \\
\hline Feb 18 BMI & 10 & 32 & 5 & 24 & 39 \\
\hline \multicolumn{6}{|l|}{ Weight } \\
\hline Pre-weight & 10 & 215 & 32 & 151 & 269 \\
\hline Dec 17 & 8 & 224 & 35 & 155 & 266 \\
\hline Jan 18 & 10 & 211 & 32 & 149 & 260 \\
\hline Feb 18 & 10 & 215 & 39 & 141 & 290 \\
\hline \multicolumn{6}{|c|}{ Fruits/Vegetables } \\
\hline Dec 01 & 5 & 2 & 2 & 0 & 5 \\
\hline Dec 08 & 5 & 3 & 2 & 1 & 5 \\
\hline Dec 15 & 8 & 3 & 2 & 0 & 5 \\
\hline Dec 22 & 5 & 3 & 2 & 0 & 5 \\
\hline Dec 29 & 6 & 2 & 2 & 0 & 5 \\
\hline Jan 19 & 7 & 3 & 2 & 1 & 5 \\
\hline Feb 16 & 7 & 3 & 1 & 1 & 5 \\
\hline \multicolumn{6}{|c|}{ Days of Exercise } \\
\hline Dec 22 & 5 & 3 & 2 & 0 & 5 \\
\hline Dec 29 & 6 & 2 & 2 & 0 & 5 \\
\hline Jan 19 & 7 & 3 & 2 & 1 & 5 \\
\hline Feb 16 & 7 & 3 & 1 & 1 & 5 \\
\hline \multicolumn{6}{|c|}{ Minutes of Exercise } \\
\hline Dec 01 & 5 & 66 & 68 & 0 & 150 \\
\hline Dec 08 & 5 & 90 & 47 & 30 & 150 \\
\hline Dec 15 & 8 & 99 & 85 & 0 & 240 \\
\hline Dec 22 & 5 & 81 & 67 & 0 & 150 \\
\hline Dec 29 & 6 & 58 & 72 & 0 & 150 \\
\hline Jan 19 & 7 & 165 & 70 & 75 & 300 \\
\hline Feb 16 & 7 & 146 & 90 & 25 & 300 \\
\hline
\end{tabular}


Table 2

Findings for Study Data $(n=10)$

\begin{tabular}{lc}
\hline \multicolumn{1}{c}{ Data Element } & $n(\%)$ \\
\hline Dropped out & $1(10 \%)$ \\
Answered phone on final follow-up call & $7(70 \%)$ \\
Lost 5\% of baseline weight & $2(20 \%)$ \\
$\qquad \begin{array}{l}\text { At End of Program }(n=7) \\
\text { Ate five servings of fruits/vegetables }\end{array}$ & $3(43 \%)$ \\
Exercised 5 days/week & $1(14 \%)$ \\
Exercised 150 minutes/week & $4(57 \%)$ \\
\hline
\end{tabular}

